\title{
Persistent Symptoms of Coronavirus Disease 2019 after Passing Acute Infection
}

\author{
HOUSSAM ELDIN H. ABD ELNABY, M.D.* and MOSTAFA A.R. HUSSEIN, M.D.** \\ The Departments of Chest Diseases* and Internal Medicine**, Faculty of Medicine, Al-Azhar University, Cairo, Egypt
}

\begin{abstract}
Background: Although the acute symptoms of COVID19 have been widely described, the longer-term effects are less well known because of the relatively short history of the pandemic. Theories attributed those symptoms to chronic inflammation (fatigue), sequelae of organ damage (pulmonary fibrosis and chronic kidney disease) and hospitalization and social isolation (muscle wasting and malnutrition).
\end{abstract}

Aim of Study: The study aimed at determining the frequency of persistent post-COVID-19 symptoms among survived COVID-19 patients, clarifying the relation between this frequency and the degree of disease severity and spotlighting some of the factors that might influence it.

Patients and Methods: This case control study was carried out during the period from March 2021 to June 2021. It included 103 subjects from the medical staff of Bab AlSha'reia University Hospital and their relatives, diagnosed 13-16 weeks ago to have COVID-19 infection by positive reverse transcription real-time polymerase chain reaction ( $\mathrm{rt}$ RT-PCR) test in their respiratory tract swabs. They were communicated either directly or through phone. Forty sex, age and body mass index (BMI) matching individuals, chosen from the medical staff of the hospital, with no history suggesting COVID-19 infection, tested negative for COVID-19 $\mathrm{IgG}$ and IgM by rapid test, served as a control group.

Results: The frequency of persistent post-COVID-19 symptoms among COVID-19 survivors was $41.75 \%$. Fatigue was the most frequently reported symptom $(38.83 \%)$, followed by dyspnoea and musculoskeletal pain (25.24\%). Fatigue, headache, musculoskeletal aches, cough and dyspnoea were significantly more frequent among hospitalized subjects compared with home-managed ones. Dyspnoea was the only symptom to show a significant frequency among ICU individuals $(p$-value $=0.015)$. As well, statistically significant higher BMI and prolonged duration of hospital stay were reported among individuals managed in the ICU when compared with the ward participants. Patients with post-COVID syndrome (PCS) were significantly older than non-symptomatic individuals ( $p$-value $<0.0001)$, with higher rates of co-morbidities $(p$-value $=0.001)$. Hypertension was the only co-morbidity that reported a discrete significant higher frequency among post-COVID-19 patients ( $p$-value $=0.003)$. Hospitalization,

Correspondence to: Dr. Houssam Eldin H. Abd Elnaby, E-Mail: drhoussam151979@gmail.com. length of hospital stay, requiring oxygen therapy and receiving either NIV or MV were all significantly linked to the developing of persistent post-COVID-19 symptoms.

Conclusion: Persistent post-COVID-19 symptoms are common among COVID-19 survivors. Older patients and those with co-morbidities (especially hypertension) are more liable to have PCS. Many factors including hospital admission, longer hospital stay, the need for oxygen therapy, NIV or MV are associated with persistent post-COVID-19 symptoms.

Key Words: Persistent symptoms - Coronavirus disease 2019.

\section{Introduction}

AFTER the severe acute respiratory syndrome (SARS) episode, some patients developed chronic fatigue syndrome/myalgic encephalomyelitis (CFS/ME) illness, which was in some cases severe enough to prevent them from returning to work for nearly 20 months [1].

Likewise, it was proposed that once an acute COVID-19 infection has been overcome, a subgroup of remitted patients are likely to experience long-term adverse effects resembling CFS/ME symptoms, such as: Persistent fatigue, diffuse myalgia, depressive symptoms and non-restorative sleep [2].

The UK National Health Service (NHS) defined the PCS as, unexplained persisting signs or symptoms over 12 weeks, developed during or after the COVID-19 infection [3]. Prolonged COVID-19 is commonly used to describe signs and symptoms that continue or develop after acute COVID-19. It includes continuous symptomatic COVID-19, called ongoing symptomatic COVID-19 (4 to 12 weeks) and post-COVID-19 syndrome (>12 weeks) [4].

Post-acute COVID-19 (also known as postCOVID syndrome and long haul syndrome) symptoms vary widely, with possibility of relapse and 
remission. The most common of them are cough, shortness of breath and fatigue. Other reported symptoms include low-grade fever, chest pain, headache, neurocognitive difficulties, muscle pain and weakness, gastrointestinal upset, rashes, metabolic disruption (such as poor control of diabetes), thromboembolic conditions, depression and other mental health conditions [5].

\section{Aim of the work:}

This study aimed at determining the frequency of persistent post-COVID-19 symptoms among survived COVID-19 patients, clarifying the relation between this frequency and the degree of disease severity and spotlighting some of the factors that might influence it.

\section{Patients and Methods}

This case control study was carried out during the period from March 2021 to June 2021. It included 103 subjects from the medical staff of Bab Al-Sha'reia University Hospital and their relatives, diagnosed 13-16 weeks ago to have COVID-19 infection by positive reverse transcription realtime PCR (rt RT-PCR) test in their respiratory tract swabs. They were communicated either directly or through phone, and appointed at Chest Outpatient Clinic, Bab Al-Sha'reia University Hospital, Cairo, Egypt. Forty sex, age and BMI matching individuals, chosen from the medical staff of the hospital, with no history suggesting COVID-19 infection, tested negative for COVID-19 IgG and IgM by rapid test, served as a control group.

Participants with any of the following were totally excluded from the study; defective mentality interfering with self-expression and communication, chronic cardiac or chest diseases, decompensated vital organ disease, medical conditions which may elicit similar symptoms to PCS, e.g. anemia, thyrotoxicosis, myxedema, neuromuscular diseases, rheumatological disorders, gastro-esophageal reflux disease (GERD), irritable bowel syndrome, familial Mediterranean fever (FMF), chronic severe rhinosinusitis, psychological troubles, pregnancy, causes of increased intracranial pressure .. etc, symptoms suggesting non-COVID-19 upper respiratory tract viral infection in the last 8 weeks.

Ethical clearance was granted by Al-Azhar Faculty of Medicine Ethics and Research Committee. Informed consents were obtained from all participants.

The data of studied population were recorded including: Name, age, sex, residence, occupation, smoking habit, smoking index and other social habits of medical importance. BMI was calculated from the formula: $\mathrm{BMI}=$ Weight $(\mathrm{kg})$ /square of height (m). Full medical history was obtained to rule out any condition included in the exclusion criteria. Thorough clinical examination was applied to exclude any temporary medical condition which may influence the study results. Complete blood count was performed (to exclude anemia).

Participants were clearly asked about studied symptoms. A Symptom was recorded as postCOVID-19 only if the patient started to complain from it during or after COVID-19 infection. When a positive reply was obtained, the individual was asked to grade his/her symptoms as mild, moderate or severe, according to the impact on normal daily activities, in which mild symptoms did not affect normal individual's activities, moderate symptoms caused little limitation, while severe symptoms obviously prevented the patient from leading his/her pre-infection ordinary life. Sharp answers only were approved, and any uncertainty was considered negative.

Long COVID-19 symptoms which are psychological in nature e.g., depression and anxiety or markedly affected by psychological status e.g., lack of concentration and sleep abnormalities were not discussed in this study, because of the fact that the incidence of such symptoms is greatly increased during pandemics, which may lead to bias, especially in the absence of a psychiatric specialist within the research team.

\section{COVID-19 Group:}

For the COVID-19 group, detailed history about the infection episode was taken including: Approximate time passed since infection, whether the patient was hospitalized or managed at home. In case of hospitalization, the patient was asked about the period of hospital stay, whether the patient was admitted to ward or ICU, the period of ICU stay if he was managed in it and history of receiving $\mathrm{O}_{2}$ therapy, non invasive ventilation (NIV) or mechanical ventilation (MV).

\section{Control Group:}

For the control group, brief history was collected to exclude possibility of previous COVID-19 infection. Screening for COVID-19 was performed using (COVID-19 IgM-IgG Rapid Test, BioMedomics Inc., Morrisville, North Carolina, USA).

\section{Statistical analysis:}

Statistical analysis of data was performed using Statistical Package for the Social Sciences (SPSS) version 18 (IBM corp., Chicago, IL, USA). Quan- 
titative variables were expressed as mean \pm SD (standard deviation) and tested using independent sample $t$-test. Qualitative variables were expressed as frequencies (percentages). Escaping the debate about the accuracy of Chi-square test in dealing with small sample size $(<5)$, and depending on the fact that exact tests could adequately used to test large sample size, we use the two-tailed Fisher's exact test to analyze qualitative data. $p$-value at the level of significance was $<0.05$.

\section{Results}

The mean age of the post-COVID-19 participants was $43.9 \pm 12.99$ years, $57.28 \%$ of them were males and $42.72 \%$ were females, with the mean $\mathrm{BMI}=28.93 \pm 5.24 \mathrm{Kg} / \mathrm{m}^{2}$. The mean period passes since onset of symptoms was $14 \pm 1.49$ weeks ( $\sim 98 \pm 7.34$ days). Non-hospitalized individuals (mild to moderate) accounted for $42.72 \%$ of the post-COVID subjects, $33.98 \%$ were admitted to ward (moderate to severe) and $23.3 \%$ needed ICU admission (critically ill), 32\% required oxygen therapy, $12.62 \%$ underwent NIV, $3.88 \%$ were mechanically ventilated, $55.34 \%$ had $\geq 1$ comorbidity(s) and $41.75 \%$ showed $\geq 1$ persistent post-COVID-19 symptom(s).

As shown in (Table 1), fatigue was the most frequently reported symptom $(38.83 \%$ ) in postCOVID-19 subjects, followed by dyspnoea and musculoskeletal pain $(25.24 \%)$, then other symptoms. Co-morbidities and all studied post-COVID19 symptoms were significantly frequent among the post-COVID-19 subjects.

Hospitalized subjects were significantly older ( $p$-value $<0.0001)$ with male predominance ( $p$ value $=0.045)$ and higher rates of co-morbidities $(p$-value $=0.001)$ when compared with nonhospitalized individuals. However, the means of BMI and time passed since acute infection showed non-significant differences between hospital admitted participants and home-managed ones. In general, persistent COVID-19 symptoms were more frequent among those who were hospitalized, but in details, fatigue, headache, musculoskeletal aches, cough and dyspnoea were significantly more frequent among hospitalized subjects compared with non-hospitalized ones ( $p$-values: $<0.0001,0.004,0.001,0.007$ and $<0.0001$ respectively), while fever, palpitation, chest pain, GIT manifestations and dysosmia were non-significantly higher among hospitalized individuals (Table 2).

As enlightened in (Table 3), no statistically significant differences were found between ward and ICU admitted subjects as regard age, sex, comorbidities, mean duration passes after acute infection and all studied symptoms except dyspnoea, which showed a significant frequency among ICU individuals ( $p$-value $=0.015)$. As well, statistically significant higher BMI and prolonged duration of hospital stay were reported among individuals managed in the ICU. ( $p$-values $=0.047$ and $<0.0001$ respectively).

Patients with PCS were significantly older than non-symptomatic post-COVID-19 individuals ( $p$ value $<0.0001$ ), with non-significant impact of sex and BMI on having persistent symptoms. PostCOVID-19 patients had a significant higher rates of co-morbidities $(p$-value $=0.001)$. However that, hypertension was the only co-morbidity that reported a discrete significant higher frequency among post-COVID-19 patients ( $p$-value $=0.003$ ). Hospitalization, length of hospital stay, requiring oxygen therapy and receiving either NIV or MV were all significantly linked to the developing of persistent post-COVID-19 symptoms. (Table 4).

Table (1): Comparing studied variables between control and post-COVID subjects.

\begin{tabular}{|c|c|c|c|c|}
\hline Variables & $\begin{array}{c}\text { Control } \\
(\mathrm{n}=40)\end{array}$ & $\begin{array}{l}\text { Post-COVID } \\
\quad(n=103)\end{array}$ & $t$ & $\begin{array}{c}p^{-} \\
\text {value }\end{array}$ \\
\hline \multicolumn{5}{|l|}{ Age (years): } \\
\hline Mean & 42.97 & 43.9 & \multirow[t]{2}{*}{0.407} & \multirow[t]{2}{*}{0.68} \\
\hline$\pm \mathrm{SD}$ & \pm 9.63 & \pm 12.99 & & \\
\hline \multicolumn{5}{|l|}{ Sex: } \\
\hline Male & $24(60 \%)$ & $59(57.28 \%)$ & & \multirow[t]{2}{*}{$0.85 \mathrm{~F}$} \\
\hline Female & $16(40 \%)$ & $44(42.72 \%)$ & & \\
\hline \multicolumn{5}{|l|}{$B M I\left(K g / m^{2}\right):$} \\
\hline Mean & 28.67 & 28.93 & \multirow[t]{2}{*}{0.267} & \multirow[t]{2}{*}{0.78} \\
\hline$\pm \mathrm{SD}$ & \pm 4.7 & \pm 5.24 & & \\
\hline \multicolumn{5}{|l|}{ Co-morbidities: } \\
\hline Yes & $13(32.5 \%)$ & $57(55.34 \%)$ & & \multirow[t]{2}{*}{$0.016 * \mathrm{~F}$} \\
\hline No & $27(67.5 \%)$ & $46(44.66 \%)$ & & \\
\hline \multicolumn{5}{|l|}{ Symptomatic: } \\
\hline Yes & $8(20 \%)$ & $43(41.75 \%)$ & & \multirow[t]{2}{*}{$0.019 * \mathrm{~F}$} \\
\hline No & $32(80 \%)$ & $60(58.25 \%)$ & & \\
\hline Fatigue & $5(12.5 \%)$ & $40(38.83 \%)$ & & $0.002 * \mathrm{~F}$ \\
\hline Headache & $3(7.5 \%)$ & $24(23.3 \%)$ & & $0.032 * \mathrm{~F}$ \\
\hline Musculoskeletal pain & $3(7.5 \%)$ & $26(25.24 \%)$ & & $0.019 * \mathrm{~F}$ \\
\hline Fever & $0(0 \%)$ & $11(10.67 \%)$ & & $0.034 * \mathrm{~F}$ \\
\hline Palpitation & $1(2.5 \%)$ & $16(15.53 \%)$ & & $0.04 * \mathrm{~F}$ \\
\hline Cough & $2(5 \%)$ & $23(22.33 \%)$ & & $0.025 * \mathrm{~F}$ \\
\hline Dyspnea & $1(2.5 \%)$ & $26(25.24 \%)$ & & $0.001 * \mathrm{~F}$ \\
\hline Chest pain & $0(0 \%)$ & $11(10.67 \%)$ & & $0.034 * \mathrm{~F}$ \\
\hline GIT manifestations & $2(5 \%)$ & $20(19.41 \%)$ & & $0.038 * \mathrm{~F}$ \\
\hline Dysosmia & $0(0 \%)$ & $11(10.67 \%)$ & & $0.034 * \mathrm{~F}$ \\
\hline$t \quad:$ Independent sam & $t$-test. & Kilogr & & \\
\hline $\mathrm{F}:$ Calculated by Fis & her's exact test. & $\mathrm{m}^{2}:$ Meter & square. & \\
\hline SD : Standard deviatio & & GIT : Gastro & -intesti & al tract. \\
\hline BMI: Body mass inde & & * : Statis & $11 y$ & gnificant. \\
\hline
\end{tabular}


Table (2): Comparing studied variables between hospitalized and non-hospitalized subjects.

\begin{tabular}{|c|c|c|c|c|}
\hline Variables & $\begin{array}{l}\text { Non-hospitalized } \\
\qquad(\mathrm{n}=44)\end{array}$ & $\begin{array}{l}\text { Hospitalized } \\
\qquad(\mathrm{n}=59)\end{array}$ & $t$ & $\underset{\text { value }}{p^{-}}$ \\
\hline $\begin{array}{l}\text { Age }(\text { years }): \\
\quad \text { Mean } \pm \text { SD }\end{array}$ & $36.88 \pm 6.84$ & $49.13 \pm 13.99$ & 5.2 & $<0.0001 * *$ \\
\hline $\begin{array}{l}\text { Sex: } \\
\quad \text { Male } \\
\text { Female }\end{array}$ & $\begin{array}{l}20(45.45 \%) \\
24(54.55 \%)\end{array}$ & $\begin{array}{l}39(66.1 \%) \\
20(33.9 \%)\end{array}$ & & $0.045^{*} \mathrm{~F}$ \\
\hline $\begin{array}{l}B M I\left(K g / m^{2}\right): \\
\quad \text { Mean } \pm \mathrm{SD}\end{array}$ & $28.47 \pm 4.6$ & $29.12 \pm 5.67$ & 0.6 & 0.54 \\
\hline $\begin{array}{l}\text { Co-morbidities: } \\
\text { Yes } \\
\text { No }\end{array}$ & $\begin{array}{l}16(36.37 \%) \\
28(63.63 \%)\end{array}$ & $\begin{array}{l}41(69.5 \%) \\
18(30.5 \%)\end{array}$ & & $0.001 * \mathrm{~F}$ \\
\hline $\begin{array}{l}\text { Time passed since onse } \\
\text { infection (weeks): } \\
\text { Mean } \\
\quad \pm \mathrm{SD}\end{array}$ & $\begin{array}{r}13.95 \\
\pm 1.41\end{array}$ & $\begin{array}{r}14.05 \\
\pm 1.55\end{array}$ & 0.3 & 0.74 \\
\hline $\begin{array}{l}\text { Symptomatic: } \\
\text { Yes } \\
\text { No }\end{array}$ & $\begin{array}{l}7(15.9 \%) \\
37(84.1)\end{array}$ & $\begin{array}{l}36(61 \%) \\
23(39 \%)\end{array}$ & & $<0.0001 * * \mathrm{~F}$ \\
\hline $\begin{array}{l}\text { Fatigue } \\
\text { Headache } \\
\text { Musculoskeletal pain } \\
\text { Fever } \\
\text { Palpitation } \\
\text { Cough } \\
\text { Dyspnea } \\
\text { Chest pain } \\
\text { GIT manifestations } \\
\text { Dysosmia }\end{array}$ & $\begin{array}{l}6(13.63 \%) \\
4(9.1 \%) \\
4(9.1 \%) \\
2(4.55 \%) \\
6(13.63 \%) \\
4(9.1 \%) \\
1(2.27 \%) \\
2(4.54 \%) \\
5(11.36 \%) \\
3(6.81 \%)\end{array}$ & $\begin{array}{l}34(57.62 \%) \\
20(33.89 \%) \\
22(37.28 \%) \\
9(15.25 \%) \\
10(16.94 \%) \\
19(32.2 \%) \\
25(42.37 \%) \\
9(15.25 \%) \\
15(25.42 \%) \\
8(13.55 \%)\end{array}$ & & $\begin{array}{l}<0.0001^{* *} \mathrm{~F} \\
0.004^{*} \mathrm{~F} \\
0.001^{*} \mathrm{~F} \\
0.11 \mathrm{~F} \\
0.78 \mathrm{~F} \\
0.007^{*} \mathrm{~F} \\
<0.0001^{*} * \mathrm{~F} \\
0.11 \mathrm{~F} \\
0.084 \mathrm{~F} \\
0.34 \mathrm{~F}\end{array}$ \\
\hline $\begin{array}{l}t: \text { Independent sam } \\
\mathrm{F}: \text { Calculated by Fis } \\
\mathrm{SD}: \text { Standard deviatio }\end{array}$ & 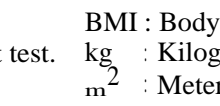 & 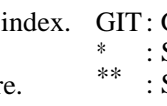 & icall & $\begin{array}{l}\text { tract. } \\
\text { ficant. } \\
\text { y significant. }\end{array}$ \\
\hline
\end{tabular}

Table (3): Comparing studied variables between ward and ICU admitted subjects.

\begin{tabular}{|c|c|c|c|c|}
\hline Variables & Ward $(n=35)$ & $\mathrm{ICU}(\mathrm{n}=24)$ & $t$ & $p$-value \\
\hline $\begin{array}{l}\text { Age (years): } \\
\quad \text { Mean } \pm \mathrm{SD}\end{array}$ & $49.51 \pm 13.15$ & $48.58 \pm 15.11$ & 0.24 & 0.8 \\
\hline $\begin{array}{l}\text { Sex: } \\
\quad \text { Male } \\
\text { Female }\end{array}$ & $\begin{array}{l}22(62.85 \%) \\
13(37.15 \%)\end{array}$ & $\begin{array}{l}17(70.83 \%) \\
7(29.17 \%)\end{array}$ & & $0.58 \mathrm{~F}$ \\
\hline $\begin{array}{r}B M I\left(K g / m^{2}\right): \\
\quad \text { Mean } \pm \mathrm{SD}\end{array}$ & $27.9 \pm 5.26$ & $30.9 \pm 5.78$ & 2.02 & $0.047 *$ \\
\hline $\begin{array}{l}\text { Co-morbidities: } \\
\text { Yes } \\
\text { No }\end{array}$ & $\begin{array}{l}21(60 \%) \\
14(40 \%)\end{array}$ & $\begin{array}{l}20(83.33 \%) \\
4(16.67 \%)\end{array}$ & & $0.084 \mathrm{~F}$ \\
\hline $\begin{array}{l}\text { Time passed since onset of } \\
\text { infection (weeks): } \\
\quad \text { Mean } \pm \mathrm{SD}\end{array}$ & $14 \pm 1.53$ & $14.12 \pm 1.58$ & 0.29 & 0.76 \\
\hline $\begin{array}{l}\text { Length of hospital stay (days): } \\
\quad \text { Mean } \pm \text { SD }\end{array}$ & $11.82 \pm 2.9$ & $18.04 \pm 4.44$ & 6.37 & $<0.0001^{* *}$ \\
\hline $\begin{array}{l}\text { Symptomatic: } \\
\text { Yes } \\
\text { No }\end{array}$ & $\begin{array}{l}20(57.14 \%) \\
15(42.86)\end{array}$ & \multicolumn{2}{|l|}{$\begin{array}{l}16(66.67 \%) \\
8(33.33 \%)\end{array}$} & $0.58 \mathrm{~F}$ \\
\hline $\begin{array}{l}\text { Fatigue } \\
\text { Headache } \\
\text { Musculoskeletal pain } \\
\text { Fever } \\
\text { Palpitation } \\
\text { Cough } \\
\text { Dyspnea } \\
\text { Chest pain } \\
\text { GIT manifestations } \\
\text { Dysosmia }\end{array}$ & $\begin{array}{l}18(51.42 \%) \\
12(34.28 \%) \\
13(37.14 \%) \\
3(8.57 \%) \\
4(11.42 \%) \\
11(31.42 \%) \\
10(28.57 \%) \\
4(11.42 \%) \\
11(31.42 \%) \\
5(14.28 \%)\end{array}$ & \multicolumn{2}{|l|}{$\begin{array}{l}16(66.67 \%) \\
8(33.33 \%) \\
9(37.5 \%) \\
6(25 \%) \\
6(25 \%) \\
8(33.33 \%) \\
15(62.5 \%) \\
5(20.83 \%) \\
4(16.67 \%) \\
3(12.5 \%)\end{array}$} & $\begin{array}{l}0.29 \mathrm{~F} \\
1.0 \mathrm{~F} \\
1.0 \mathrm{~F} \\
0.13 \mathrm{~F} \\
0.28 \mathrm{~F} \\
1.0 \mathrm{~F} \\
0.015^{*} \mathrm{~F} \\
0.46 \mathrm{~F} \\
0.23 \mathrm{~F} \\
1.0 \mathrm{~F}\end{array}$ \\
\hline $\begin{array}{l}t \quad: \text { Independent sample } t \text {-test. } \\
\mathrm{F} \text { : Calculated by Fisher's exact test. } \\
\mathrm{SD}: \text { Standard deviation, }\end{array}$ & $\begin{array}{l}\text { ICU : Inten } \\
\text { BMI : Bod } \\
\mathrm{kg}: \text { Kilo } \\
\mathrm{m}^{2}: \text { Mete }\end{array}$ & $\begin{array}{ll}\text { are unit. } & \text { GIT } \\
\text { index. } & * \\
& * * \\
\text { re, } & \end{array}$ & $\begin{array}{l}\text { : Gastro-int } \\
\text { : Statisticall } \\
\text { : Statisticall }\end{array}$ & $\begin{array}{l}\text { l tract. } \\
\text { hificant. } \\
\text { hly significant }\end{array}$ \\
\hline
\end{tabular}


Table (4): Comparing studied variables between patients with persistent symptoms and non-symptomatic patients.

\begin{tabular}{|c|c|c|c|c|}
\hline Variables & $\begin{array}{l}\text { Patients with persistent } \\
\text { symptoms }(n=43)\end{array}$ & $\begin{array}{l}\text { Non-symptomatic } \\
\text { patients }(n=60)\end{array}$ & $t$ & $\begin{array}{c}p^{-} \\
\text {value }\end{array}$ \\
\hline $\begin{array}{l}\text { Age (years): } \\
\quad \text { Mean } \pm \text { SD }\end{array}$ & $53.72 \pm 13.24$ & $36.86 \pm 6.75$ & 8.36 & $<0.0001 * *$ \\
\hline $\begin{array}{l}\text { Sex: } \\
\quad \text { Male } \\
\text { Female }\end{array}$ & $\begin{array}{l}22(51.16 \%) \\
21(48.84 \%)\end{array}$ & $\begin{array}{l}36(60 \%) \\
24(40 \%)\end{array}$ & & $0.42 \mathrm{~F}$ \\
\hline $\begin{array}{r}B M I\left(\mathrm{~kg} / \mathrm{m}^{2}\right): \\
\quad \text { Mean } \pm \mathrm{SD}\end{array}$ & $29.68 \pm 5.8$ & $28.39 \pm 4.72$ & 1.22 & 0.22 \\
\hline $\begin{array}{l}\text { Co-morbidities: } \\
\text { Yes } \\
\text { No }\end{array}$ & $\begin{array}{l}32(74.41 \%) \\
11(25.59 \%)\end{array}$ & $\begin{array}{l}25(41.67 \%) \\
35(58.33 \%)\end{array}$ & & $0.001 * \mathrm{~F}$ \\
\hline $\begin{array}{l}\text { Hypertension } \\
\text { DM } \\
\text { Obesity } \\
\text { CKD } \\
\text { CLD }\end{array}$ & $\begin{array}{l}23(53.48 \%) \\
11(25.59 \%) \\
20(46.51 \%) \\
4(9.3 \%) \\
3(6.97 \%)\end{array}$ & $\begin{array}{l}15(25 \%) \\
7(11.67 \%) \\
16(26.67) \\
1(1.67 \%) \\
0(0 \%)\end{array}$ & & $\begin{array}{l}0.003 * \mathrm{~F} \\
0.11 \mathrm{~F} \\
0.058 \mathrm{~F} \\
0.15 \mathrm{~F} \\
0.06 \mathrm{~F}\end{array}$ \\
\hline $\begin{array}{l}\text { Time passed since infection } \\
\text { (weeks): } \\
\text { Mean } \pm \text { SD } \\
\text { Hospitalized patients }\end{array}$ & $\begin{array}{l}14.09 \pm 1.47 \\
36(83.72 \%)\end{array}$ & $\begin{array}{l}13.95 \pm 1.51 \\
23(38.33 \%)\end{array}$ & 0.47 & $\begin{array}{l}0.63 \\
<0.0001 * * \mathrm{~F}\end{array}$ \\
\hline $\begin{array}{l}\text { Length of hospital stay (days): } \\
\quad \text { Mean } \pm \text { SD }\end{array}$ & $15.55 \pm 4.42$ & $12.47 \pm 4.57$ & 2.52 & $0.014 *$ \\
\hline $\begin{array}{l}\text { Length of ICU stay (days): } \\
\quad \text { Mean } \pm \text { SD }\end{array}$ & $11.93 \pm 4.17$ & $10.12 \pm 4.59$ & 0.92 & 0.3635 \\
\hline $\begin{array}{l}\text { Patients required O2 therapy } \\
\text { Patients received NIV } \\
\text { Patients received MV }\end{array}$ & $\begin{array}{l}23(53.48 \%) \\
12(27.9 \%) \\
4(9.3 \%)\end{array}$ & $\begin{array}{l}10(16.67 \%) \\
1(1.67 \%) \\
0(0 \%)\end{array}$ & & $\begin{array}{l}0.0001 * * \mathrm{~F} \\
<0.0001 * * \mathrm{~F} \\
0.027 * \mathrm{~F}\end{array}$ \\
\hline $\begin{array}{ll}t & : \text { Independent sample } t \text {-test. } \\
\mathrm{F} & : \text { Calculated by Fisher's exact } \\
& \text { test. } \\
\mathrm{SD} & : \text { Standard deviation. } \\
\text { BMI } & : \text { Body mass index. }\end{array}$ & $\begin{array}{ll}\mathrm{kg} & : \text { Kilogram. } \\
\mathrm{m} 2 & : \text { Meter square. } \\
\mathrm{DM} & : \text { Diabetes mellitus. } \\
\mathrm{CKD} & : \text { Chronic kidney disease. } \\
\text { CLD } & : \text { Chronic liver disease. }\end{array}$ & $\begin{array}{l}\text { ICU : Intensive care unit. } \\
\text { O2 : Oxygen. } \\
\text { NIV : Non-invasive ventilation. } \\
\text { MV : Mechanical ventilation. }\end{array}$ & $\begin{array}{l}* \\
* *\end{array}$ & $\begin{array}{l}\text { ly significant. } \\
\text { ly highly } \\
\text { t. }\end{array}$ \\
\hline
\end{tabular}

In (Table 5) it was clarified that, less than 7\% of patients with persistent post-COVID-19 symptoms had a single symptom, $18.6 \%$ had $2-3$ symptoms, while more than $74 \%$ had four or more symptoms. On the other hand, more than two thirds of the symptomatic patients $(67.44 \%)$ grade their symptoms as moderate, less than quarter of them $(23.26 \%)$ grade their symptoms as severe, while only $9.3 \%$ grade their symptoms as mild.

Table (5): Distribution of number and grades of symptoms among patients with persistent symptoms.

\begin{tabular}{lcccc}
\hline Variables & \multicolumn{4}{c}{ Patients with persistent symptoms $(\mathrm{n}=43)$} \\
\hline Number of & 1 & $2-3$ & $4-5$ & $>5$ \\
symptoms & $3(6.98 \%)$ & $8(18.6 \%)$ & $18(41.86 \%)$ & $14(32.56 \%)$ \\
Grades of & Mild & Moderate & Severe & \\
symptoms & $4(9.3 \%)$ & $29(67.44 \%)$ & $10(23.26 \%)$ & \\
\hline
\end{tabular}

\section{Discussion}

The findings recorded by this study are close to a French one included 120 subjects, with mean age of $63.2 \pm 15.7$ years, $20 \%$ admitted to ICU, investigated 110 days after hospital admission. In that study fatigue was the leading symptom, being presented in $55 \%$ of assessed individuals, dyspnoea came second (42\%), then cough in $16.7 \%$, anosmia in $13.3 \%$ and chest pain in $10.8 \%$ [6].

Our results are relatively matching with those of a study conducted in Rome, Italy, included 143 individuals, with mean age of $56.5 \pm 14.6$ years, assessed $60.3 \pm 13.6$ days after hospital discharge, among whom $53.8 \%$ needed supplemental $\mathrm{O}_{2}$ $12.6 \%$ admitted to ICU, $14.7 \%$ needed NIV and $4.9 \%$ underwent MV. Patients with $\geq 1$ symptom(s) represented $87.4 \%$ of the study population and $55.2 \%$ of the included subjects had $\geq 3$ symptoms at the time of assessment. Fatigue was manifested in $53.1 \%$ of studied subjects, dyspnoea in $43.4 \%$, worsened quality of life in $44.1 \%$, joint pain in $27.3 \%$ and chest pain in $21.7 \%$ [7].

Following a mean period of $71 \pm 17$ days after onset of COVID-19 symptoms, Llach and his colleagues recorded inappropriate sinus tachycardia in $17 \%$ of their 200 post-COVID-19 patients with 
negative history of previous structural heart disease nor electricity defect. This finding agree a lot with that revealed by our study [8] .

Alongside, a Norwegian study with an equal number of subject to ours, including $100 \%$ exinpatients, assessed 3 months after hospital admission, revealed dyspnoea in $52 \%$ of the study personnel [9].

In a study focused only on discussing postCOVID-19 fatigue, Townsend and his colleagues reported fatigue in 67 patients out of his 128 participants $(52.3 \%)$, after a period of 10 weeks from hospital discharge/or last acute symptom [10]. As well, a study recorded fatigue in $69 \%$ of its 124 subjects, after 3 months of hospital discharge [11] On the other hand an Iranian study revealed chronic fatigue syndrome in only $17.5 \%$ among 120 previous COVID-19 infections, 6 months after hospital discharge [12].

Another Scandinavian study, included 180 participants, with a vast majority of outpatients (95.6\%), performed 125 days after symptom onset, showed heterogeneous results to those of the current study; $55 \%$ of subjects had $\geq 1$ symptom(s), $28.9 \%$ with fatigue, anosmia in $27.2 \%$, dyspnoea in $8.3 \%$, headache in $7.2 \%$, myalgia in $7.2 \%$, nausea in $6.1 \%$, cough in $4.4 \%$ and diarrhoea in $4.4 \%$ [13]

Two smaller Chinese studies with post-COVID19 participants in different degrees of disease severity, conducted 3 months after hospital discharge, revealed variable results. The first one included 76 subjects, and showed some similarity to our results; chest tightness and palpitations in $62 \%$ of studied individuals, dyspnoea in $61 \%$, cough in $60 \%$, fatigue in $59 \%$ and fever in $20 \%$ [14], while the other, included 60 individuals, 55\% of them had post-COVID syndrome, and muscle pain was the only symptom to show a frequency almost the same as ours (25\%) [15].

However, the results of another Italian study performed 4 months after hospital discharge on 238 post-COVID subjects, with a median age of 61 years, in which $72.3 \%$ needed supplemental $\mathrm{O} 2,11.8 \%$ admitted to ICU and $8.8 \%$ needed MV, were extremely lower than ours, as arthralgia and myalgia were reported in $5.9 \%$ of studied subjects, dyspnoea in $5.5 \%$, ageusia, anosmia, cough, diarrhoea and chest pain in $\leq 5 \%$ [16]. Another large study with 4182 post-COVID-19 individuals reported a surprisingly low frequency of PCS (2.3\% after $>12$ weeks from symptom onset) [17]
Unlike our findings, a Japanese small study (63 subjects) recorded lower frequencies for fatigue and cough (9.5\% and $6.3 \%$ respectively), although that, it revealed a matching frequency for dysomnia (9.7\%) [18]. Parallel to the Japanese study Augustin et al., reported fatigue in $9.7 \%$, dyspnoea in $8.6 \%$ and anosmia in $12.4 \%$ among his 442 post-COVID subjects, followed up 4 months after symptom onset [19].

Likewise, persistent post-COVID-19 symptoms were found in $38.7 \%$ of the 434 outpatients subjects of a study held in Norway, with a median of 117 days after symptom onset. That study revealed dyspnoea in $15 \%$ of participants, smell dysfunction in $12 \%$, taste dysfunction in $10 \%$, myalgia in $8.5 \%$, headache and cough in $6 \%$ and GIT symptoms in $\leq 5 \%$ [20]

At the same line, the frequency of PCS reported by a study with 114 previously hospitalized participants, having a mean age of $54 \pm 12$ years, were by far lesser than ours, with only $14 \%$ and $6.1 \%$ of the study population had dyspnoea and cough respectively 175 days after onset of symptoms [21] This huge gap may be attributed to the very long interval between acute illness and the assessment for persistent symptoms in that study, which almost equals double of ours.

In contrary to the previous study, a large Chinese study included 1733 individuals, all of them were previously inpatients, with a median age of 57 years, in which $67.6 \%$ needed supplemental $\mathrm{O}_{2}$ and $4 \%$ admitted to ICU, recorded PCS in $76 \%$ of the study population, after a median period of 186 days after symptom onset, with fatigue and muscle weakness equally occupied the first rank as the most frequent symptoms (63\%), dyspnoea in the third position (26\%), taste disorder came late (7\%) and lastly, chest pain and headache $(\leq 5 \%)$ [22].

On the other hand, our findings were much lower those found by Dennis and co-workers, who investigated 201 subjects $(81.6 \%$ of them were outpatients), with a mean age of $44 \pm 11$ years, after a median period of 141 days after symptom onset. The vast majority in that study $(99 \%)$ had $\geq 4$ symptoms at the time of assessment and $42 \%$ had $\geq 10$ symptoms by the same time. Fatigue was recorded in $98 \%$ of subjects, dyspnoea in $87.1 \%$, muscle aches in $86.7 \%$ and headache in $82.6 \%$ were the most frequently reported, followed by joint pain in $78.1 \%$, fever in $75.1 \%$, cough in $73.6 \%$, chest pain in $73.1 \%$ and diarrhoea in $59.2 \%$ [23]. 
Exceeding our findings by far, a study with 100 post-COVID-19 individuals, investigated in a mean period of 93 days after infection, with 75 of them not-hospitalized during the novel virus acute infection, revealed fatigue in $80 \%$ of participants and respiratory symptoms in 59\% [24].

The great variety among studies from different countries or even within the same country could be explained by the recurrent mutations of COVID19 , as it is thought that those mutations did not only affect the infectivity of the virus, but also influenced the long haul syndrome symptoms and severity.

In spite of the numerous studies explored the frequencies of PCS and its components, few studies were interested in comparing this frequencies among different categories e.g., hospitalized versus non-hospitalized, ward versus ICU, ... etc.

In a large study included 112 previously hospitalized COVID-19 subjects and 2001 nonhospitalized individuals (345 confirmed COVID19 patients, 882 symptom-based COVID-19 patients and 774 suspected COVID-19 patients) analyzed in a mean period of $79 \pm 17$ after onset of symptoms, the comparison of confirmed COVID19 patients (hospitalized versus confirmed nonhospitalized subjects), revealed no statistically significant differences between both groups as regard the frequency of fatigue, headache, dyspnoea, anosmia, aguesia, nausea and diarrhoea. Hospitalized individuals showed significant higher rates of cough, vomiting and low-grade fever ( $p$ values $=0.02,0.01$ and $<0.0001$ respectively), while non-hospitalized confirmed COVID-19 subjects showed significant higher rates of chest tightness, muscular pain, high-grade fever and palpitation ( $p$-values $=0.02,0.0006,0.004$ and 0.003 respectively). These results meet ours in some points and oppose ours in others. The same study recorded that the proportion of women and the proportion of patients without pre-existing co-morbidities were lower in the subjects of hospitalized sample, who were older $(p$-values $=<0.001,<0.001$ and 0.007 respectively), which completely agree with our observations. However, in contrary to our findings, the study reported a significant higher BMI in the hospitalized group [25]

Our results agree with those of a study included 100 COVID-19 survivors discharged from a large University Hospital, assessed 4-8 weeks postdischarge by a multidisciplinary team of rehabilitation professionals, as they reported a significant higher frequency of breathlessness among the ICU group in comparison with the ward group $(65.6 \%$ versus $42.6 \%)$ with a $p$-value $=0.03$ [26].

An Asian study on 355 participants, corresponds to our findings that patients with severe COVID19 illness (need oxygen therapy, NIV or MV) and those who required a prolonged time of clinical improvement (have more duration of hospital stay) are more liable to develop PCS ( $p$-values $=0.02$ and 0.001 respectively). However, the same study object ours by detecting an association between PCS and female sex [27]

The results of the current work also match with those of an Egyptian study, included 425 participants and concluded that the main determinants of the persistent post-COVID-19 symptoms were the need for oxygen therapy ( $p$-value $<0.001$ ), preexisting hypertension $(p$-value $=0.039)$ and any chronic co-morbidity ( $p$-value $=0.004)$ [28]. Alongside, Tenforde and his colleagues accord with us that the presence of any chronic condition affects the rapid return to basal health status $(p$-value $=$ 0.03) [29].

Finally, Garrigues et al., mismatch with our results, as their comparison between ward and ICU post-COVID-19 subjects led to non-statistically significant differences regarding studied symptoms and parameters [6].

\section{Conclusion:}

Older patients and those with co-morbidities (especially hypertension) are more liable to have post-COVID syndrome. Hospital admission, longer hospital stay, the need for oxygen therapy, NIV or MV are all significantly linked to the developing of persistent post-COVID-19 symptoms.

\section{References}

1- MOLDOFSKY H. and PATCAI J.: Chronic widespread musculoskeletal pain, fatigue, depression and disordered sleep in chronic post-SARS syndrome; a case-controlled study. BMC Neurology, 11: 1-7, 2011.

2- PERRIN R., RISTE L. and HANN M.: Into the looking glass: Post-viral syndrome post COVID-19. Med. Hypotheses., 144: 110055, 2020.

3- NATIONAL INSITUTE for HEALTH and CARE EXCELLENCE: Management of the long-term effects of COVID19. NICE: London, UK, 1-7, 2020.

4- UK OFFICE for NATIONAL STATISTICS: Prevalence of ongoing symptoms following Coronavirus (COVID19) infection in the UK. ONS; London, UK, 2021.

5- DASGUPTA A., KALHAN A. and KALRA S.: Long term complications and rehabilitation of COVID-19 patients. J. Pak. Med. Assoc., 70: S131-135, 2020.

6- GARRIGUES E., JANVIER P., KHERABI Y., et al.: Postdischarge persistent symptoms and health-related quality 
of life after hospitalization for COVID-19. J. Infect., 81 (6): e4-e6, 2020.

7- CARFI A., BERNABEI R. and LANDI F.: Persistent symptoms in patients after acute COVID-19. JAMA, 324 (6): 603-605, 2020

8- LLACH J.A., BAZAN V., LLADOS G., ADELINO R., DOMINGUEZ M.J., MASSANELLA M., BISBAL F., SARRIAS A., BAYES-GENIS A., MATEU L. and SABATE R.V.: Inappropriate sinus tachycardia in post COVID-19 syndrome. EP Europace., 23 (8): iii 126, 2021.

9- LERUM T.V., AALØKKEN T.M., BRØNSTAD E., et al.: Dyspnoea, lung function and CT findings three months after hospital admission for COVID. Eur. Respir. J., 57(4): 2003448, 2021

10- TOWNSEND L., DYER A.H., JONES K., et al.: Persistent fatigue following SARS-CoV-2 infection is common and independent of severity of initial infection. PLOS ONE, 15 (11): e0240784, 2020.

11- VAN DEN BORST B., PETERS J.P., BRINK M., SCHOON Y., et al.: Comprehensive health assessment three months after recovery from acute COVID-19. Clin. Infect. Dis., ciaa1750, 2020

12- SIMANI L., RAMEZANI M., DARAZAM I.A., et al.: Prevalence and correlates of chronic fatigue syndrome and post-traumatic stress disorder after the outbreak of the COVID-19. J. Neurovirol., 27 (1): 154-159, 2021.

13- PETERSEN M.S., KRISTIANSEN M.F., HANUSSON K.D., et al.: Long COVID in the Faroe Islands - a longitudinal study among non-hospitalized patients. Clin. Infect. Dis., ciaa1792, 2020.

14- LIANG L., YANG B., JIANG N., et al.: Three-month Follow-up Study of Survivors of Coronavirus Disease 2019 after Discharge. J. Korean Med. Sci., 35 (47): e418, 2020.

15- LU Y., LI X., GENG D., et al.: Cerebral micro-structural changes in COVID-19 patients - An MRI-based 3-month follow-up study. E. Clinical Medicine, 25: 100484, 2020.

16- BELLAN M., SODDU D., BALBO P.E., BARICICH A., et al.: Respiratory and psychophysical sequelae among patients with COVID-19 four months after hospital discharge. JAMA Netw. Open, 4 (1): e2036142, 2021.

17- SUDRE C.H., MURRAY B., VARSAVSKY T., et al.: Attributes and predictors of long COVID. Nat Med., 27 (4): 626-631, 2021

18- MIYAZATO Y., MORIOKA S., TSUZUKI S., et al.: Prolonged and late-onset symptoms of coronavirus disease 2019. Open Forum Infect. Dis., 7 (11): ofaa507, 2020.
19- AUGUSTIN M., SCHOMMERS P., STECHER M., DEWALD F., et al.: Post-COVID syndrome in nonhospitalized patients with COVID-19: A longitudinal prospective cohort study. Lancet Reg. Health Eur., 6: 100122, 2021

20- STAVEM K., GHANIMA W., OLSEN M.K., et al.: 1.56 months after COVID-19 in non-hospitalized subjects: A population-based cohort study. Thorax., 76 (4): 405 407, 2021.

21- HAN X., FAN Y., ALWALID O., et al.: Six-month followup chest CT findings after severe COVID-19 pneumonia. Radiology, 299 (1): E177-E186, 2021.

22- HUANG C., HUANG L., WANG Y., et al.: 6-month consequences of COVID-19 in patients discharged from hospital: A cohort study. The Lancet., 397 (10270): 220232, 2021

23- DENNIS A., WAMIL M., ALBERTS J., et al.: Multiorgan impairment in low-risk individuals with post-COVID-19 syndrome: A prospective, community-based study. BMJ Open, 11 (3): e048391, 2021.

24- VANICHKACHORN G., NEWCOMB R., COWL C.T., MURAD M.H., et al.: Post COVID-19 syndrome (long haul syndrome): description of a multidisciplinary clinic at the Mayo Clinic and characteristics of the initial patient cohort. Mayo. Clin. Proc., 96 (7): 1782-1791, 2021.

25- GOËRTZ Y.M.J., VAN HERCK M., DELBRESSINE J.M., VAES A.W., et al.: Persistent symptoms 3 months after a SARS-CoV-2 infection: The post-COVID-19 syndrome? ERJ Open Res., 6 (4): 00542, 2020.

26- HALPIN S.J., MCLVOR C., WHYATT J., ADAMS A., et al.,: Postdischarge symptoms and rehabilitation needs in survivors of COVID-19 infection: A cross-sectional evaluation. J. Med. Virolo., 93 (2): 1013-1022, 2021

27- MAHMUD R., RAHMAN M., RASSEL M.F., BINTE MONAYEM F., et al.: Post-COVID-19 syndrome among symptomatic COVID-19 patients: A prospective cohort study in a tertiary care center of Bangladesh. PLOS ONE, 16 (4): e0249644, 2021.

28- GALAL I, HUSSEIN A.A.R.M., AMIN M.T., SAAD M.M., et al.: Determinants of persistent post-COVID-19 symptoms: Value of a novel COVID-19 symptom score. Egyptian Journal of Bronchology, 15: 10, 2021.

29- TENFORDE M.W., KIM S.S., LINDSELL C.J., et al.: Symptom duration and risk factors for delayed return to usual health among outpatients with covid-19 in a multistate health care systems network-United States, MarchJune 2020. MMWR, 69 (30): 993-998, 2020. 


\section{الأعراض المستمرة لمرض فيروس كورونا بعد تجاوز العدوى الحمادة فيرون}

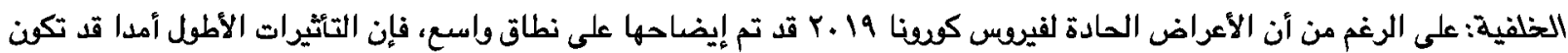

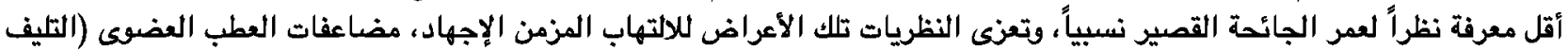

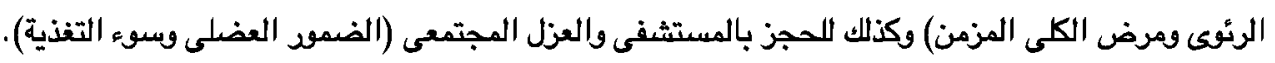

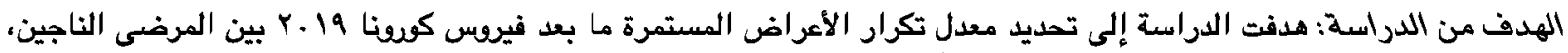

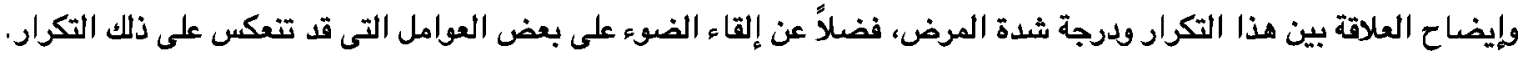

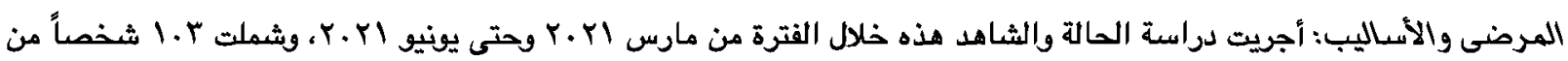

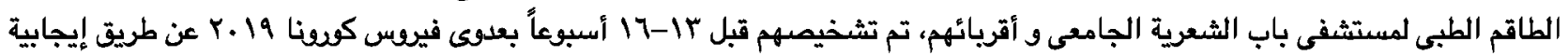

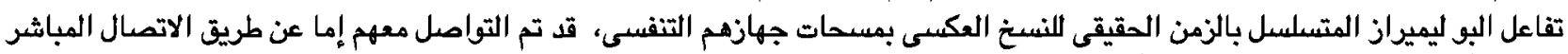

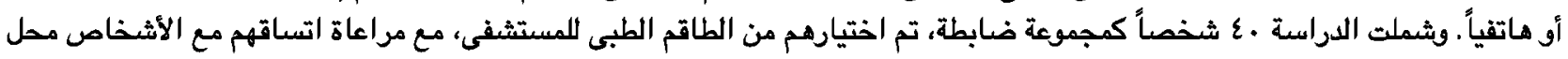

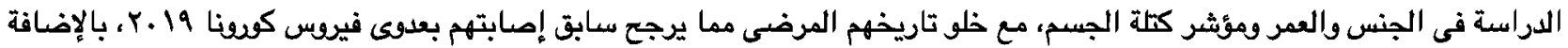

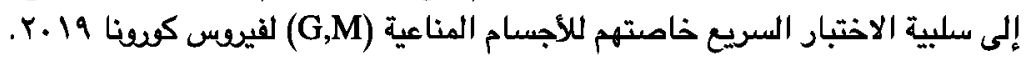

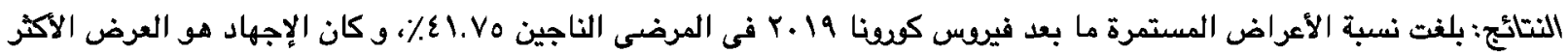

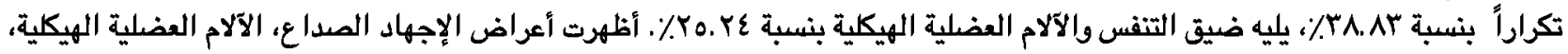

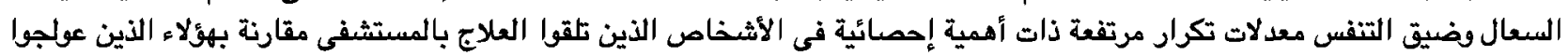

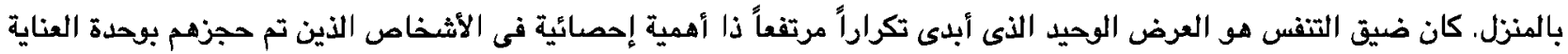

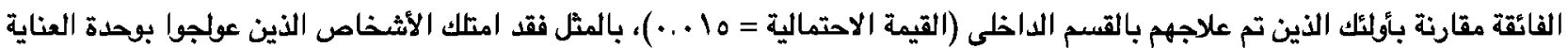

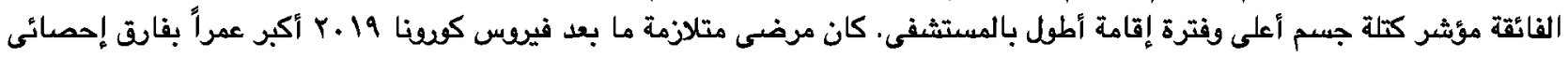

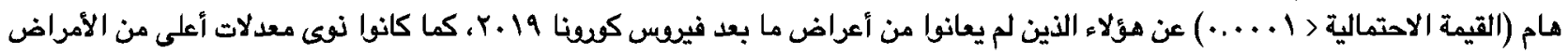

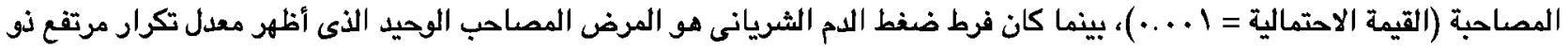

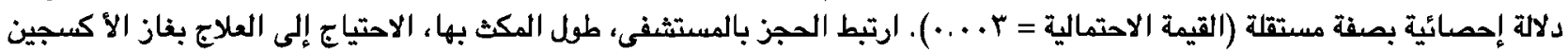

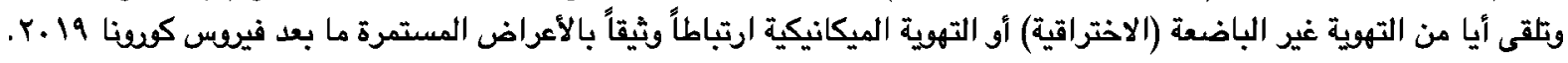

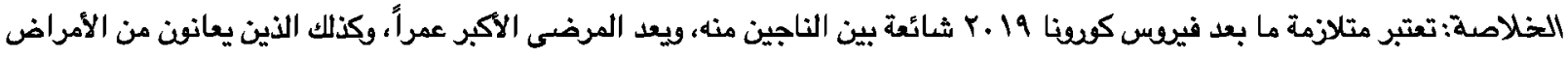

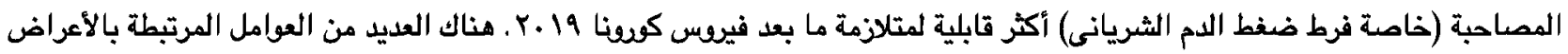

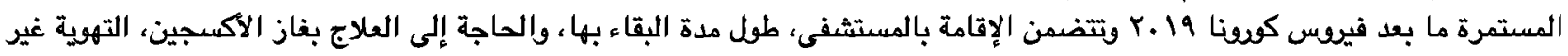
الباضعة أو التهوية الميكانيكية. 